\title{
Finite Element Modeling of Crack Generation in Laser Shock Peening Processed Airfoils
}

\author{
Fang Li, ${ }^{1,2}$ Xue Qi, ${ }^{1}$ and Dan Xiang ${ }^{1,3}$ \\ ${ }^{1}$ Intelligent Automation Inc., 15400 Calhoun Drive, Suite 190, Rockville, MD 20855, USA \\ ${ }^{2}$ Department of Mechanical Engineering, New York Institute of Technology, Old Westbury, NY 11568, USA \\ ${ }^{3} X$-wave Innovations, Inc., 555 Quince Orchard Road, Suite 500-B, Gaithersburg, MD 20878, USA
}

Correspondence should be addressed to Fang Li; fli08@nyit.edu

Received 28 March 2014; Accepted 10 September 2014; Published 13 October 2014

Academic Editor: Gang Liu

Copyright (C) 2014 Fang Li et al. This is an open access article distributed under the Creative Commons Attribution License, which permits unrestricted use, distribution, and reproduction in any medium, provided the original work is properly cited.

\begin{abstract}
Laser shock peening (LSP) is a surface treatment process for airfoils that is achieved by the induction of compressive stress. While LSP is a mature and reliable surface treatment process, slight anomalies during the process, or variations in material ductility and geometries, may cause unintended formation of small subsurface cracks in the resultant LSP processed material. In this study, we developed a 3D FEM model to simulate the formation and predict the sizes of cracks generated by inappropriate LSP processing in airfoil specimens in order to avoid producing such subsurface cracks. The Johnson-Cook plastic material model along with the consideration of effects of high strain rate was used to describe the plasticity of Ti alloys. The constants in this plastic model have been optimized with experimental data. The FEM model also includes both damage initiation and evolution criteria to simulate cracks generated by LSP process in the specimens. The simulated crack sizes and locations in the specimens have been validated by the experimental results.
\end{abstract}

\section{Introduction}

Laser shock peening (LSP) is a surface treatment method applied to metallic materials for fatigue enhancement [1-4], in which laser energy is converted into shock waves at the surface to induce compressive residual stress. During an LSP process, the area on the test subject that will be treated is locally covered with two types of overlays: a black coating that is opaque to the laser beam and a thin film of water that is transparent to the laser beam. When the laser beam strikes the surface of the opaque overlay, the coating layer vaporizes into plasma. The water traps the thermally expanding vapor, which causes a sudden and high-pressure shock wave that propagates into the material [5]. LSP process has many advantages over other surface treatment methods, including deep compressive residual stress, low cold work amplitudes, and no physical contact. It has been widely used for surface treatment of various metallic materials. For example, LSP has been used to treat foreign object damage- (FOD-) prone areas such as the leading edge of compressor airfoils in modern gas turbines and can significantly enhance their fatigue life and strength [6].

While LSP is a promising manufacturing process for the fatigue life extension of metallic materials, inappropriate processing conditions, low material ductility, and certain geometry can cause unintended small subsurface cracks in the resultant LSP processed material, which will result in a reduction of fatigue life [7-9]. In general, cracks are more likely to form in thin metal parts because the shock wave in thick materials attenuates to the point where only elastic strain continues. In contrast, the shock wave in thin materials may not have attenuated below the dynamic yield strength of the materials before reaching the back surface of the test sample. The reflected tensile wave has large amplitude, which can potentially cause cracks in materials. It is especially true for thin sections less than $12 \mathrm{~mm}$, where a split laser pulse is required to impact on opposite sides to balance the generated forces [1]. The cracks are more likely to occur in the middle plane of a specimen since a large tensile stress is generated due to the overlapping of two reflected tensile waves. 
TABLE 1: Parameters for Johnson-Cook damage initiation model.

\begin{tabular}{lcccc}
\hline$d_{1}$ & $d_{2}$ & $d_{3}$ & $d_{4}$ & $d_{5}$ \\
\hline-0.09 & 0.14 & 0.5 & 0.014 & 3.87 \\
\hline
\end{tabular}

For the LSP process, the laser pulse needs to be optimized in order to maximize the efficiency of production while minimizing the chance of subsurface crack formation, especially for thin components such as airfoils in aircrafts that need LSP treatment [1]. However, obtaining optimal parameters through experiments is both time-consuming and expensive because LSP processing is a very costly experimental method. Therefore, it is necessary to develop a theoretical model to simulate the crack generation during LSP processing and to provide guidance of choosing appropriate processing parameters. Developing an accurate and reliable simulation method is challenging because LSP is a very high strain rate process [6]. In the past few years, several axisymmetric and 3D finite element models have been developed to simulate the residual stress profile by the LSP process [9-13]. However, the effects of high strain rate on the material plasticity were not fully considered in most of the simulation models. Recently, Amarchinta et al., from Wright State University, developed an inverse optimization-based methodology to create a plastic material model and simulated the residual stresses induced by the LSP process $[6,14]$. The effects of high strain rate on the material plasticity have been considered in their study. However, only the residual stress profiles induced by LSP were considered, and the damage model for crack generation was not included in their simulation.

In this study, we developed a 3D model to simulate the crack formation in LSP-processed Ti-6246 (Ti-6Al-2Sn-4Zr$6 \mathrm{Mo}$ ) airfoil-like specimens. The Johnson-Cook (JC) model was used to describe material plasticity and material fracture properties were described by JC damage initiation and evolution criteria in the 3D FEM model (Table 1). Because JC plastic constants for Ti-6246 at high strain rate are unrecorded in literatures, an inverse optimization-based methodology was used to obtain those constants in this study. The experimental procedures are as follows. A 2D axisymmetric FEM model was developed to simulate residual stress profiles in LSPprocessed Ti-6246 specimens. Johnson-Cook (JC) plastic constants were optimized by fitting the simulated residual stress profiles with experimental profiles measured on Ti6246 (the experimental data was provided by LSP Technologies, Inc.). The model for crack generation was developed, tested, and validated through the experimental data of cracks in LSP-processed airfoil-like specimens under two different processing conditions (two laser spot sizes). To ensure the crack initiation, two types of specimens were fabricated with simultaneous double-sided LSP processing under two different laser spot sizes. The cracks in LSP-processed samples were quantified with high-frequency acoustic microscopy. The JC parameters for damage evolution were optimized by comparing simulated cracks with those in experiments for large-spot processed samples, and then, the developed FEM model was applied to the small-spot processed samples.

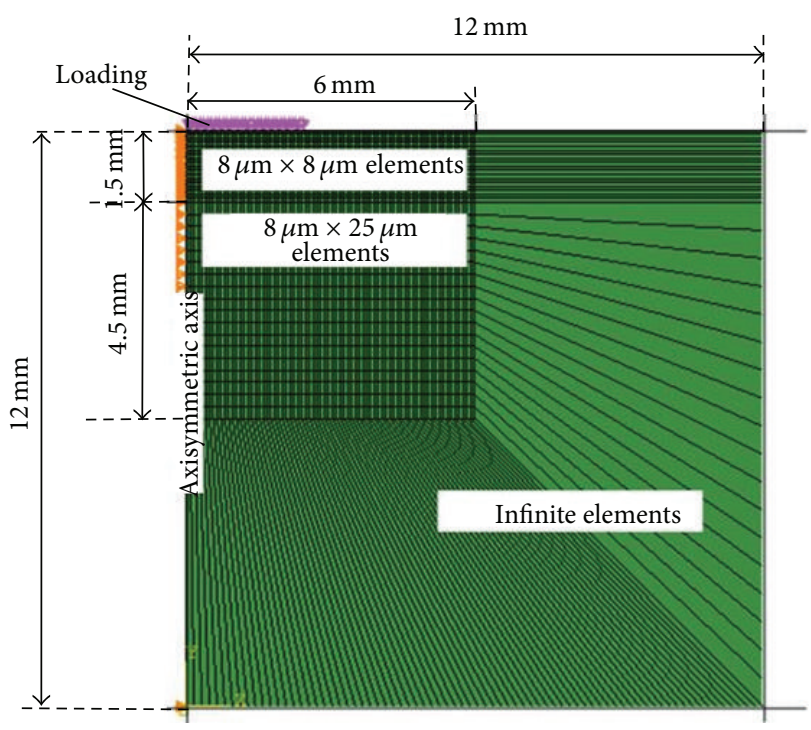

FIgURE 1: The finite element model for thick flat plate.

By quantitative comparison, experimental results were well consistent with the simulated data.

This paper is organized as follows. Section 2 describes the details of two FEM models, including the geometry, loading, material models, and analysis steps, as well as the optimization method of JC plastic constants. Section 3 introduces the experiment of crack detection with acoustic microscopy. Section 4 presents and discusses the simulation results, including optimization of JC plastic constants, calibration of damage evolution parameters, and simulation of crack formation in airfoil coupons. Finally, the conclusion remarks are stated in Section 5.

\section{Finite Element Modeling (FEM)}

\subsection{FEM Model for Thick Flat Plates}

2.1.1. Geometry and Loading. A thick flat plate was chosen as the geometry model for optimization of JC plastic constants due to the following two reasons: (1) the thick flat plate can be precisely described with a $2 \mathrm{D}$ axisymmetric model, which significantly saves computation time; (2) for simulation of residue stress profiles in LSP-processed thick plate specimens, fracture material model is not necessary to be included in FEM model because usually cracks do not form in a thick plate. However, fracture model has to be included in the case of an airfoil-like structure since cracks are likely to be generated in it. In this study, the geometry of FEM model was defined according to the experiments in which thick flat plates made of Ti-6246 were treated by LSP with a laser spot size of $5.6 \mathrm{~mm}$ in diameter to induce residual stress.

Figure 1 shows the axisymmetric finite element model for a thick flat plate, which can be treated as a half-infinite space. In order to balance the accuracy and computational efficiency, the region close to the loading surface was meshed with refined elements, and the further region was meshed with coarse elements. The radius and the depth of the finely 


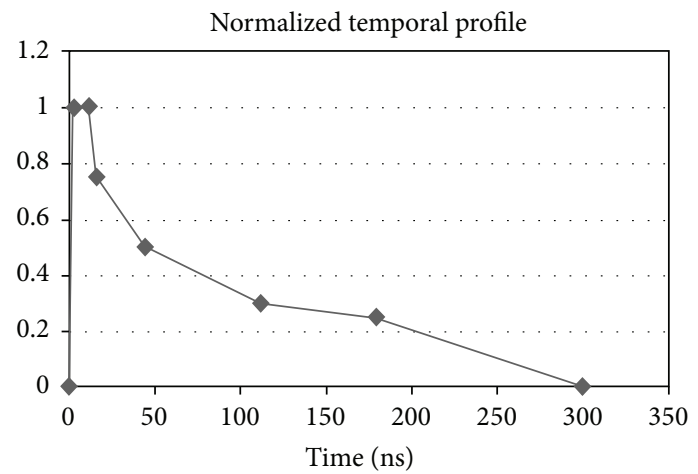

(a)

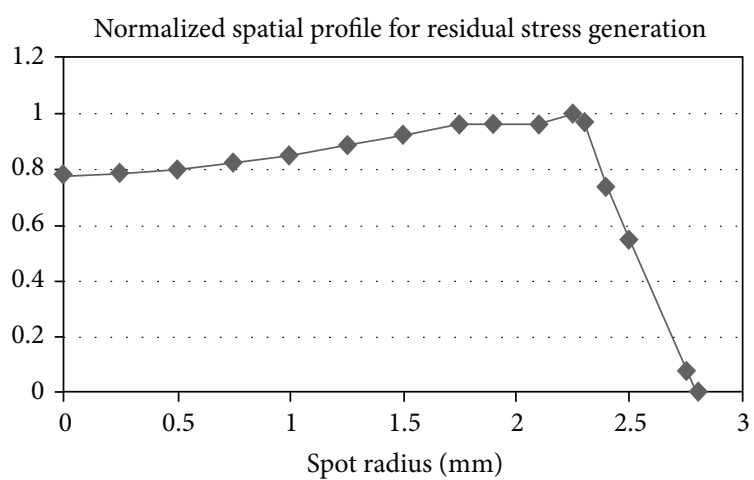

(b)

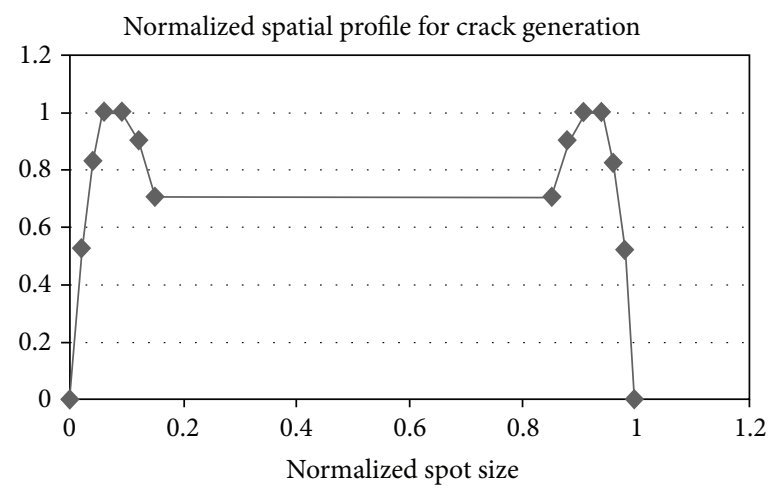

(c)

FiguRE 2: (a) The normalized temporal profile, (b) normalized spatial profile of the shock wave for residual stress generation, and (c) normalized spatial profile of the shock wave for crack generation.

meshed portion were a little more than twice the radius of the laser spot. Infinite elements were assigned on the side and bottom boundaries to eliminate reflected waves. According to convergence study, a refined mesh $(8 \mu \mathrm{m} \times 8 \mu \mathrm{m})$ was assigned to a $6 \mathrm{~mm} \times 1.5 \mathrm{~mm}$ rectangular area under the top surface. Below that, a coarse mesh $(8 \mu \mathrm{m} \times 25 \mu \mathrm{m})$ was defined to facilitate computations.

According to the experimental condition, the normalized loading profile applied in the FEM model was described in Figures 2(a) and 2(b). The full width at half maximum (FWHM) of the pressure pulse was 45 ns. Three different values of peak pressure were used: $6.7 \mathrm{GPa}, 5.3 \mathrm{GPa}$, and 4.2 $\mathrm{GPa}$.

2.1.2. Material Model. The Ti-6246 used in this model was assumed to be ideally homogeneous and isotropic material. Young's modulus, Poisson's ratio, and density of Ti-6246 were $113 \mathrm{GPa}, 0.33$, and $4460 \mathrm{~kg} \mathrm{~m}^{-3}$, respectively. The material plasticity was defined with a JC plastic model, which is widely used to represent high strain rate phenomena. The strain rate hardening, strain hardening, and thermal softening effects on the plastic deformation are simply incorporated in the JC model as a multiplication form and expressed as follows [15]:

$$
\sigma=\left(A+B \varepsilon^{n}\right)\left[1+C\left(\ln \frac{\dot{\varepsilon}}{\dot{\varepsilon}_{0}}\right)\right]\left[1+D\left(\frac{T-T_{0}}{T_{0}}\right)^{m}\right]
$$

where $\sigma$ is the stress; $\varepsilon$ is the plastic strain; $A$ is the initial yield stress; $\dot{\varepsilon}_{0}$ is the reference strain rate; $T_{0}$ is the reference temperature; and $B, n, C, D$, and $m$ are the undetermined coefficients. In this simulation, the temperature effect was ignored because the increase in temperature caused by laser shock is minimal. Therefore, the constant $D$ was assumed to be zero. The other five constants $\left(A, B, C, n\right.$, and $\left.\dot{\varepsilon}_{0}\right)$ were optimized with an inverse optimization-based method which will be described in the later section.

2.1.3. Analysis Steps. The simulation of residual stress profile consisted of two phases. The first phase was the laser firing phase, which spanned a very short interaction time as the shock waves traveled through the component. The second phase was the recovery process, in which stable mechanical equilibrium occurred relatively slow. ABAQUS/Explicit was used to simulate the high-speed dynamic process in the first phase. The total time selected for this step was $4000 \mathrm{~ns}$ based on our convergence study. The explicit algorithm is not computationally suitable for the second phase due to its very short time increments. Therefore, ABAQUS/Standard was used in the second phase to achieve stable equilibrium.

2.1.4. Optimization of the JC Plastic Constants. To the best of our knowledge, the JC constants for Ti 6246 at high strain rate are not available in literatures. Therefore, to obtain the 


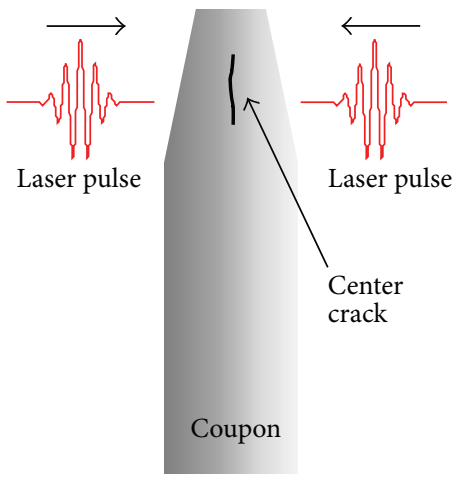

(a)

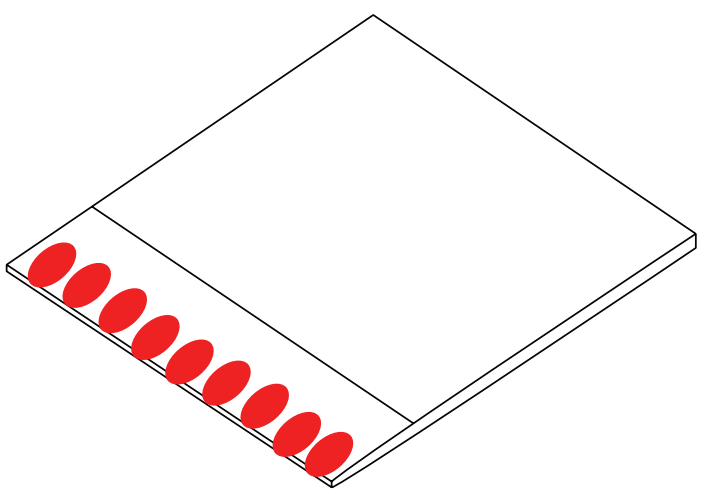

(b)

FIGURE 3: (a) Schematic of the two-sided peening process; (b) pattern of the laser spots along the tapered edge of the coupon.

JC constants for Ti 6246 is essential prior to simulating LSP-processed crack generation with FEM model. Recently, Amarchinta et al. developed an inverse optimization-based methodology to predict residual stress profiles in Ti-6Al-4V using the JC plasticity model [6]. Experimental residual stress data for one set of LSP process parameters was applied to optimize the JC plastic constants. The optimized constants were then tested with data for other processing conditions.

Here, we followed a similar method to optimize JC constants for Ti 6246. Least squares curve fitting was conducted to minimize the error between the residual stress profiles from FEM simulations and experiments. Values for the JC constants $A, B, n, C$, and $\varepsilon_{0}$ were obtained for $\mathrm{Ti}$ 6246 by using the following optimization procedure: (1) residual stress profiles induced by LSP were simulated in ABAQUS with the JC plasticity model; (2) the ABAQUS simulation was repeatedly called by MATLAB with different JC constants. For every call, the least squares error (LSE) between simulation and experimental data was calculated by the following equation:

$$
e=\sqrt{\sum_{\text {thickness }}\left(\mathrm{RS}_{\mathrm{exp}}-\mathrm{RS}_{\mathrm{sim}}\right)^{2}}
$$

where $\mathrm{RS}_{\text {exp }}$ and $\mathrm{RS}_{\text {sim }}$ were the experimental and simulated residual stress, respectively. The JC model constants were modified until the minimal value of LSE was found. The experimental residual stress depth profiles were determined using the X-ray diffraction method by LSP Technologies, Inc. The measured residual profile was an average value over an area of $2 \mathrm{~mm}$ by $5 \mathrm{~mm}$ square within the laser spot.

\subsection{FEM Model for Airfoil Coupons}

2.2.1. Geometry and Loading. The FEM model was developed based on the coupon dimension and loading profiles used in the experiments. As shown in Figure 3, the airfoil coupon had a tapered edge reassembling the leading edge of an airfoil. The high-energy laser pulse was split into two beams, which were simultaneously incident to the working piece from both sides and the laser peened spots were separated by $1 \mathrm{~mm}$. Two elliptical spot sizes were employed: one was $6.5 \times 4 \mathrm{~mm}$, and the other was $3.25 \times 2 \mathrm{~mm}$. The centers of the spots were $3.2 \mathrm{~mm}$ away from the thinner edge for the large spot and $1.6 \mathrm{~mm}$ for the small spot. The normalized experimental loading profiles in the space and time domains are shown in Figures 2(a) and 2(c). The peak pressure was $7.54 \mathrm{GPa}$. In the model, in order to save computation time and memory, only a small portion of the airfoil coupon associated with a laser peened spot was simulated. Therefore, the model was defined with an elliptical shape. Figures 4(a) and 4(b) show the dimensions of the model for large spot and small spot-processed coupons. Symmetric boundaries were applied to midplanes so that only one-quarter of the plate was simulated. In order to mesh the structure appropriately, the coupon was partitioned as shown in Figure 4. Since the pressure was not uniformly distributed, the loading area had to be partitioned into several regions. Within each region, the loading amplitude was a constant. The mesh size in the model was set as $25 \mu \mathrm{m}$ (fine adjusted with the program) as shown in Figure 4(c).

2.2.2. Material Model. The material model for airfoil coupons was the same as the one described in Section 2.1 in terms of density, elasticity, and plasticity. In addition, in order to simulate crack formation, a JC damage initiation criterion and a damage evolution model were included. Both of them are available in ABAQUS. In general, a ductile material undergoes damage in two forms: the softening of the flow stress and degradation of the elastic modulus [16]. In Figure 5, the stress-strain relationship including the onset and evolution of damage is illustrated with the solid curve. The dashed curve shows the relationship in the absence of damage. In the JC damage initiation criterion, the equivalent plastic strain at the onset of damage $\bar{\varepsilon}_{0}^{\mathrm{pl}}$ is given by

$$
\begin{aligned}
\bar{\varepsilon}_{0}^{\mathrm{pl}}= & {\left[d_{1}+d_{2} \exp \left(-d_{3} \sigma^{*}\right)\right]\left[1+d_{4} \ln \left(\frac{\bar{\varepsilon}^{\mathrm{pl}}}{\dot{\varepsilon}_{0}}\right)\right] } \\
& \times\left(1+d_{5} T^{*}\right),
\end{aligned}
$$




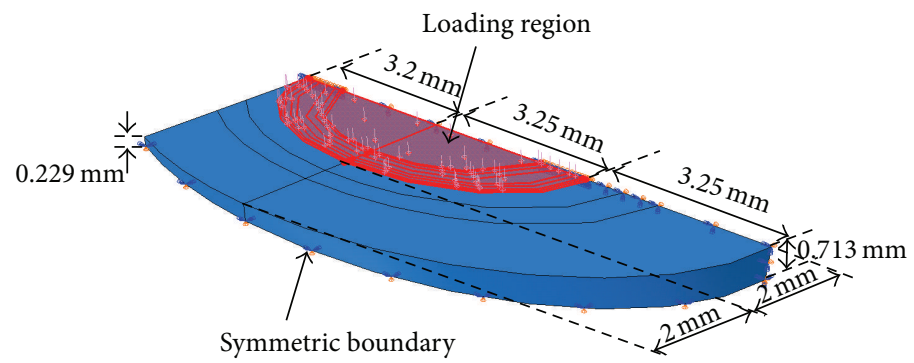

(a)

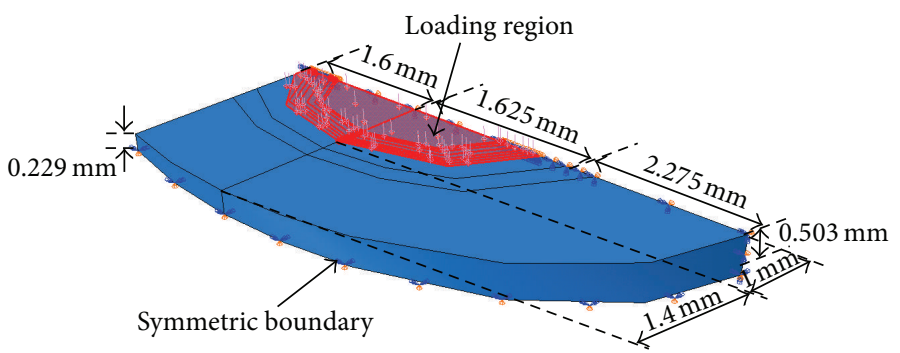

(b)

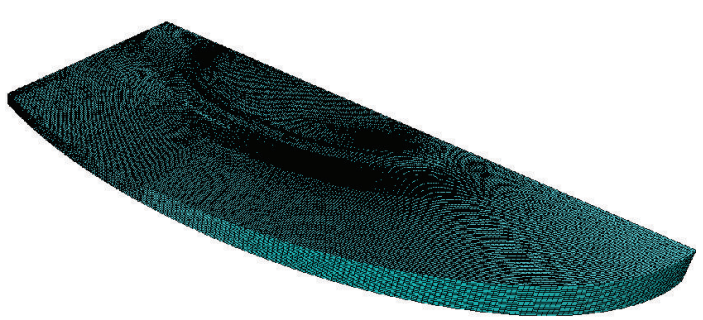

(c)

Figure 4: The dimensions of the 3D finite element model for (a) large spot processed and (b) small spot processed coupons. (c) Meshed model for large spot processed coupons.

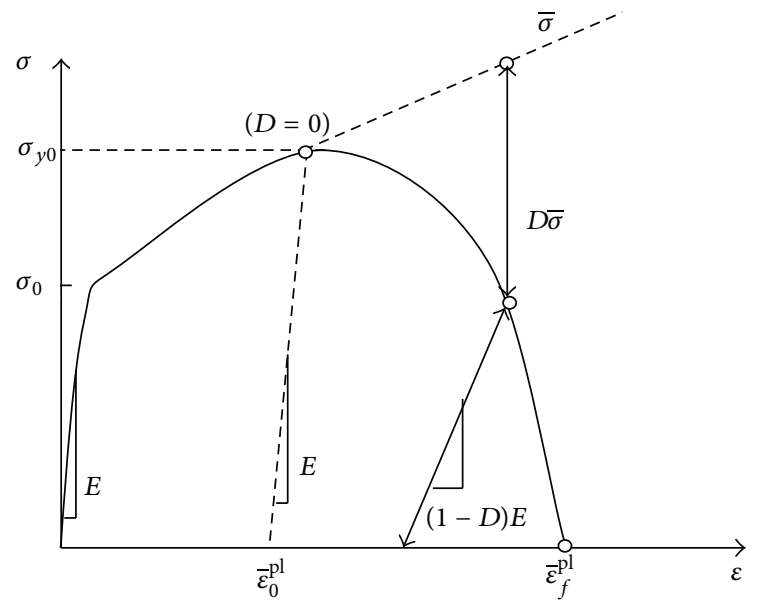

FIGURE 5: Stress-strain curve with progressive damage degradation (ABAQUS Analysis User's Manual).

where $\sigma^{*}$ is the dimensionless pressure-stress ratio

$$
\sigma^{*}=\frac{\sigma_{m}}{\bar{\sigma}}
$$

$\sigma_{m}$ is the average of the three normal stresses, and $\bar{\sigma}$ is the Von Mises equivalent stress. $\bar{\varepsilon}^{\mathrm{pl}}$ and $\dot{\varepsilon}_{0}$ are plastic strain rate and reference strain rate, respectively. $T^{*}$ is the homologous temperature. $d_{1} d_{2}, d_{3}, d_{4}$, and $d_{5}$ represent different failure parameters. Fracture initiates when the accumulated incremental effective plastic strains exceed $\bar{\varepsilon}_{0}^{\mathrm{pl}}$. Similar to the JC plastic model, the damage initiation criteria did not take note of temperature effects. Since the failure parameters in the JC damage initiation criteria for Ti-6246 are not available in literatures, the parameters for Ti-64 were used in this study, which was assumed to be similar to those of Ti-6246 [17].

In this simulation, after damage initiation, the damage evolution was based on the effective plastic displacement $\bar{\mu}^{\mathrm{pl}}$. The damage variable, $D$, increases according to a linear form

$$
\dot{D}=\frac{\dot{\mu}^{\mathrm{pl}}}{\bar{\mu}_{f}^{\mathrm{pl}}} .
$$

In this equation, $\dot{D}$ is the damage variable rate and $\bar{\mu}^{\mathrm{pl}}$ is the effective plastic displacement rate. At the beginning of damage initiation $\bar{\mu}^{\mathrm{pl}}$ is equal to 0 and later the damage initiation is given by $\bar{\mu}^{\mathrm{pl}}=L \bar{\varepsilon}^{\mathrm{pl}}$ where $L$ is the characteristic length of the element or the mesh size, which is about $25 \mu \mathrm{m}$ in this model. $\bar{\mu}_{f}^{\mathrm{pl}}$ is the effective plastic displacement at the point of failure (the formation of a local crack). Full degradation happens when $D$ reaches 1 (Figure 5). Therefore, the effective plastic displacement at the point of failure is an important factor that determines the failure point. The smaller the value of the effective plastic displacement is, the more easily the crack forms. This parameter was optimized with experimental data of occurrence of cracking.

For cracking simulations, the loading profiles in the space and time domains used in the model were the same as those used in the experiments. The JC damage initiation and evolution models were incorporated with the JC plasticity model for these simulations. Only the ABAQUS/Explicit module was employed to simulate this high-speed dynamic process, and an analysis time of 4000 ns was used. 


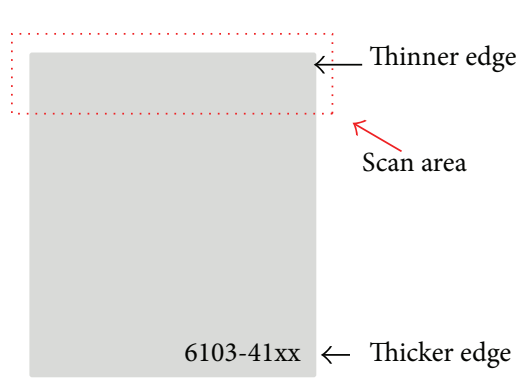

(a)

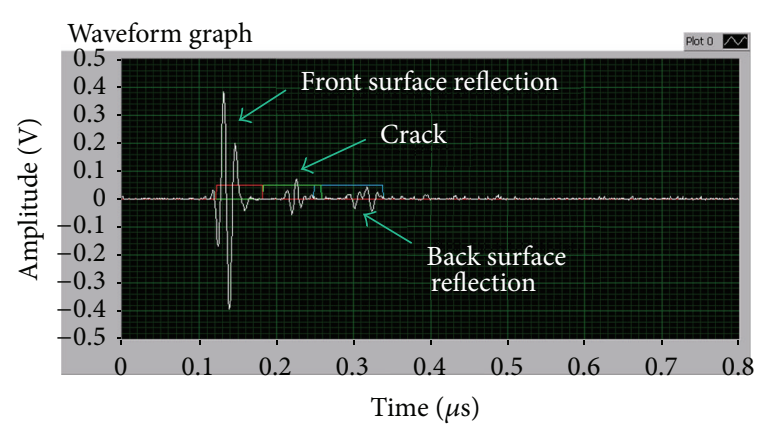

(b)

FIgURE 6: (a) Scanning area by acoustic microscopy; (b) a typical waveform of scanning point at a cracking position.

\section{Experiments of Crack Detection with Acoustic Microscopy}

Acoustic microscopy employs very high or ultra-high-frequency ultrasound with a high spatial resolution for nondestructive evaluation. It can penetrate most of solid materials and generate images of internal features, including defects such as cracks, delaminations, and voids. In this study, a custom-designed acoustic microscope was used to quantify the occurrence of LSP-induced cracks in LSP-processed samples. The acoustic microscope contains an $x-y-z$ translation stage with a resolution of 1 micron. An ultrasonic transducer that was attached to the stage was used to interrogate the sample. A high sampling rate digitizer $\left(2 \mathrm{GSa} \mathrm{S}^{-1}\right)$ was used to digitize the detected waveforms. Application software with a graphic user interface (GUI) was developed to provide the controls for transducer/stage transition, scan area, scan resolution, and display of acquired time waveform. The display of processed image was integrated into the GUI interface. In order to obtain a high resolution, a $100 \mathrm{MHz}$ transducer and a $300 \mathrm{MHz}$ bandwidth pulser/receiver were used in this study. During the scan, both the transducer and the coupon were placed in distilled water. The LSP processed area was scanned as indicated by the red dotted rectangle in Figure 6(a). Figure 6(b) shows the typical A-scan waveforms by the acoustic microscopy. With such a highfrequency transducer, the front and back echoes are widely separated, and acoustic reflections at the crack interface can be clearly identified. A signal gate, between the front surface reflection and back surface reflection, was selected to monitor the occurrence of cracks. The amplitude of gated signal was used to produce the scanned images. With the use of acoustic microscopy, the crack size, orientation, depth, and so forth can be measured with great accuracy.

\section{Results and Discussions}

4.1. Optimization of JC Constants in Plastic Material Model. The residual stress profile was calculated with the thick plate model discussed earlier. JC constants were fitted based on the experimental residual stress profile data from the experiment with a peak pressure of $6.7 \mathrm{GPa}$. In the optimization, the FEM model was called a total of 120 times and the LSE was reduced from $432 \mathrm{MPa}$ to $300 \mathrm{MPa}$ by curve fitting. Figure 7 shows the comparison of simulated and experimental residual stress profiles for three LSP processing conditions. The simulated data were obtained with optimized JC constants: $A=130.98 \mathrm{MPa}$, $B=6835.6 \mathrm{MPa}, n=0.36465, C=0.0062$, and $\varepsilon_{0}=$ $1.8488 \mathrm{~s}^{-1}$. The LSEs are $300 \mathrm{MPa}, 343 \mathrm{MPa}$, and $410 \mathrm{MPa}$, respectively, which are the minimal values obtained. The simulated profiles are very consistent with only small discrepancies compared with the experimental ones by X-ray measurements. The discrepancies may have been induced by the errors in LSP processing parameters and X-ray measurements. Optimized parameters were used in the following simulations.

4.2. Optimization of the Effective Plastic Displacement at the Failure Point in the Damage Evolution Model. Figure 8 shows the typical experimental crack images for two spot sizes produced by in-house high-frequency acoustic microscopy. The thinner edge of the coupon is along the $x$-axis. The dashed line shows the outline of the laser spot and the solid lines show the nominal crack locations. Two concentrated regions were found to have cracks that were induced by a large laser spot (Region 1: along the circumference of a $5.4 \times$ $3 \mathrm{~mm}$ ellipse with its center coincident with that of the laser spot; Region 2: around $1.5 \mathrm{~mm}$ away from the $y$-axis (two sides) and around $0.5-1 \mathrm{~mm}$ away along the thinner edge of the sample). As shown in Figure 8(b), cracks located in two regions for small laser spots were found. The first one is $0.7-$ $1.5 \mathrm{~mm}$ away from the thinner edge, and the second one is 2.0-2.5 mm away from the thinner edge. Cracks in both of these two regions were less than $0.5 \mathrm{~mm}$ away from the $y$-axis (two sides).

The effective plastic displacement coefficient for damage evolution modeling was optimized, with the coupon model, by comparing the simulated crack locations in the large spotprocessed coupons with the experimental results. Figure 9 shows the simulated crack locations close to the middle plane. Different values of the effective plastic displacement coefficient were used for each simulation. The loading area is a $6.25 \times 4 \mathrm{~mm}$ ellipse with the elliptical center $3.2 \mathrm{~mm}$ away from the thinner edge, as shown in Figure 9(a). The locations of the thinner edges and the loading areas in Figures 

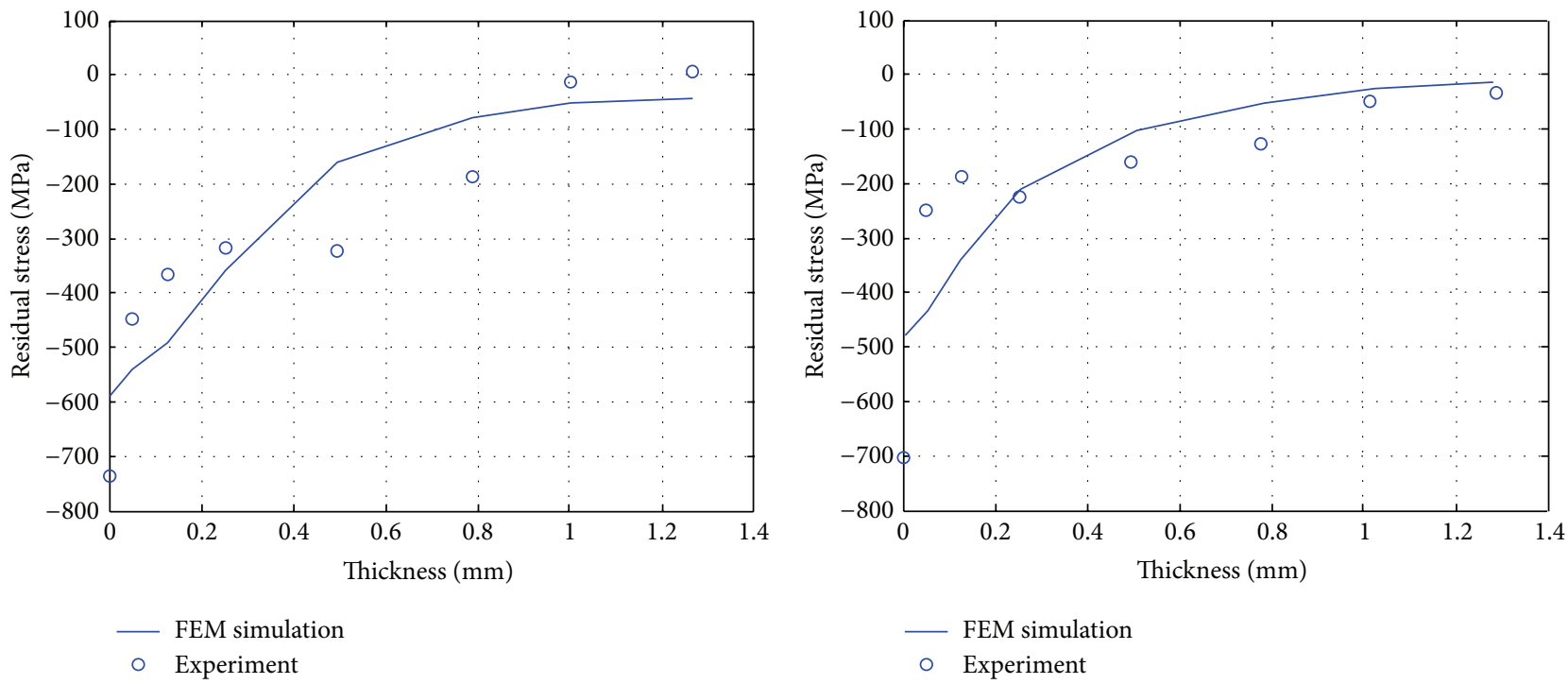

(a)

(b)

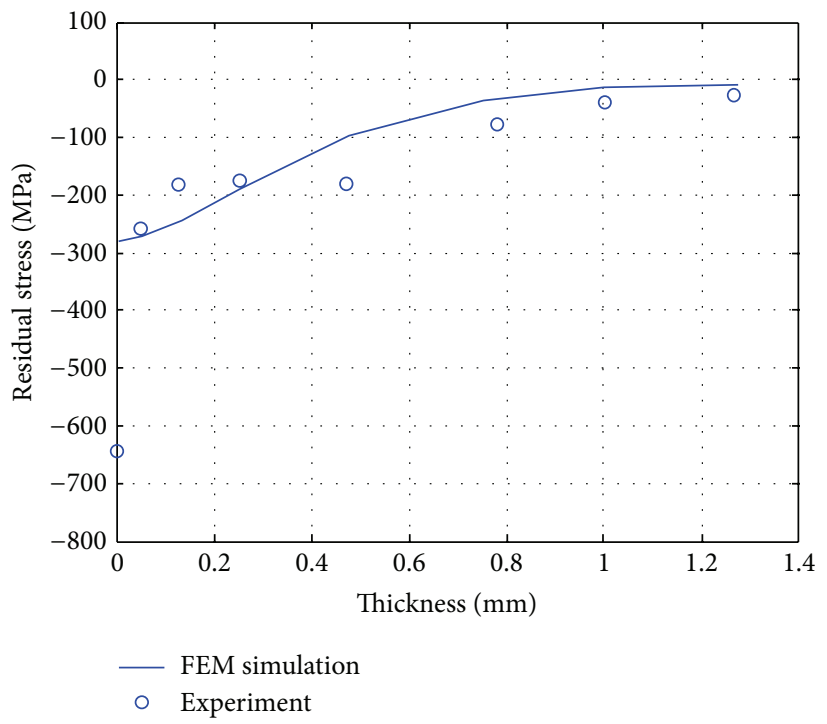

(c)

FIGURE 7: Simulated residual stress profiles with optimized JC constants $(A=130.98 \mathrm{MPa}, B=6835.6 \mathrm{MPa}, n=0.36465, C=0.0062$, and $\varepsilon_{0}=1.8488 \mathrm{~s}^{-1}$ ) at (a) $6.7 \mathrm{GPa}$; (b) $5.3 \mathrm{GPa}$; (c) $4.2 \mathrm{GPa}$.

9(b) to 9 (d) are the same as those shown in Figure 8(a). It was found that the cracks were located in three regions: (1) along the circumference of a $5.4 \times 3 \mathrm{~mm}$ ellipse with its center coincident with that of the loading; (2) around $0.5 \mathrm{~mm}$ away from the thinner edge and $1.3 \mathrm{~mm}$ from the symmetric axis of the loading; and (3) along the thinner edge. The simulated crack locations are similar to the experimental observations shown in Figure 8(a), in terms of regions 1 and 2. In addition, with decreasing values of the effective plastic displacement coefficient, the length for crack occurrence along the circumference of the $5.4 \times 3 \mathrm{~mm}$ ellipse increases.

To optimize the effective plastic displacement at the failure point, the relationship between the effective plastic displacement coefficient and the distance for cracking $(L)$, along the circumference of the ellipse, was established, as shown in Figure 10. According to the experimental median value of the cracking length along the circumference of the ellipse (from 48 cracks), which is around $1.8 \mathrm{~mm}$, the value of the effective plastic displacement at the failure point was calibrated to be $0.42 \mu \mathrm{m}$.

4.3. Simulated Crack Generation by Small Laser Shot Spots. With the optimized parameters from the large spot results, the 3D FEM model was tested by simulating the crack generation for a coupon processed with small spots. Figure 11 displays the simulated crack locations close to the middle plane of the coupon. The simulation results showed that the 


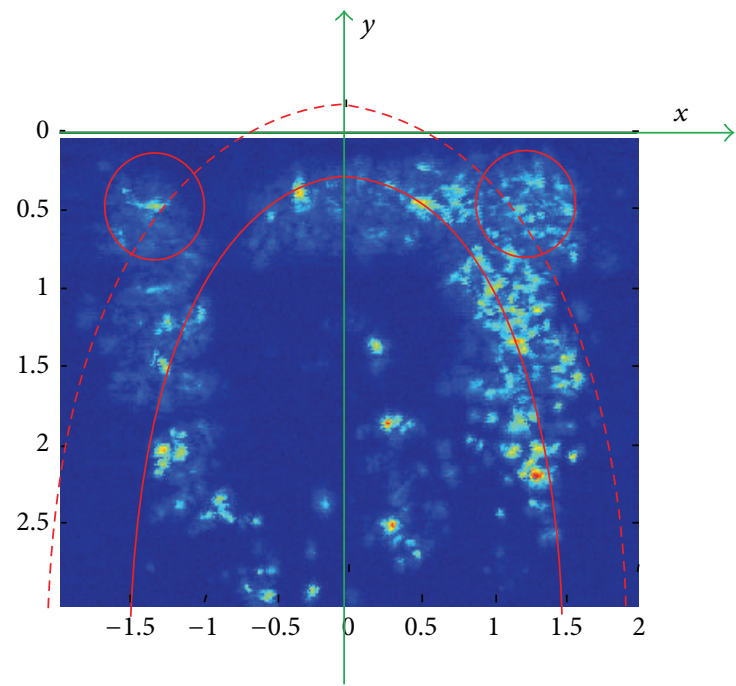

(a)

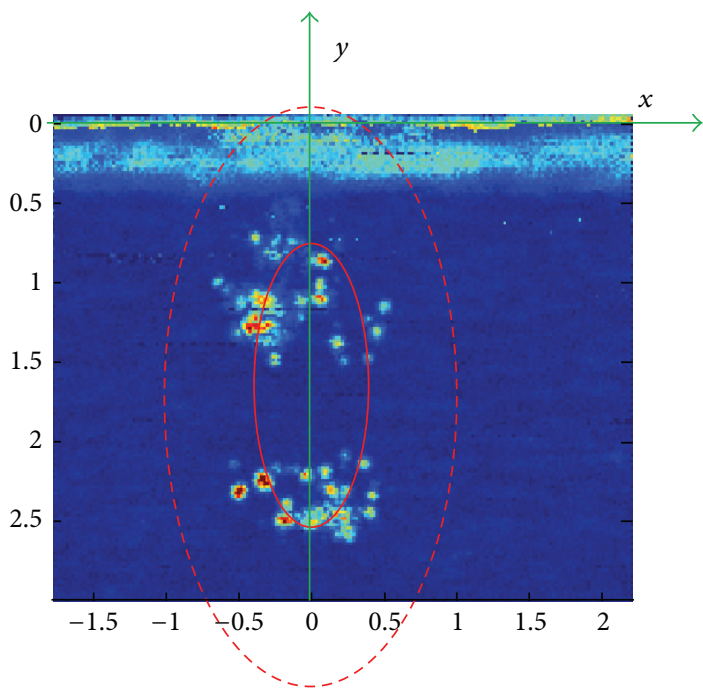

(b)

FIGURE 8: Typical crack images produced by (a) large laser spot and (b) small laser spot. The crack is measured by high-frequency acoustic microscopy. The dashed line shows the laser spot and the solid lines show the region where cracks locate.

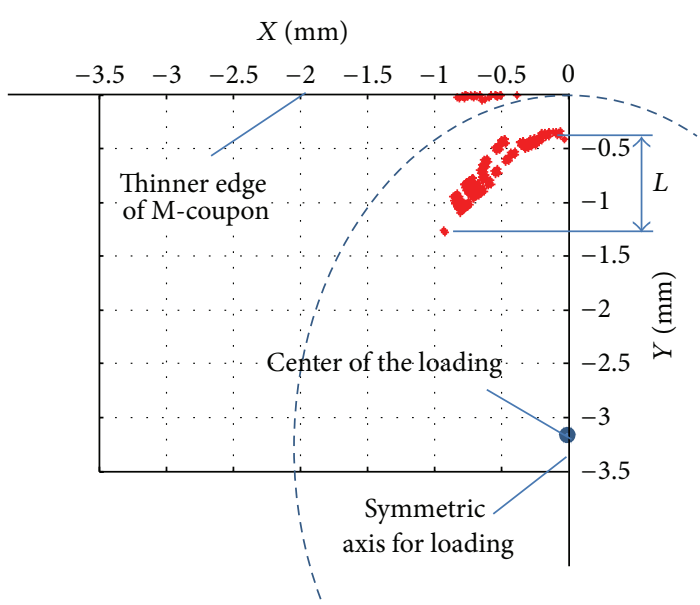

(a)

$X(\mathrm{~mm})$

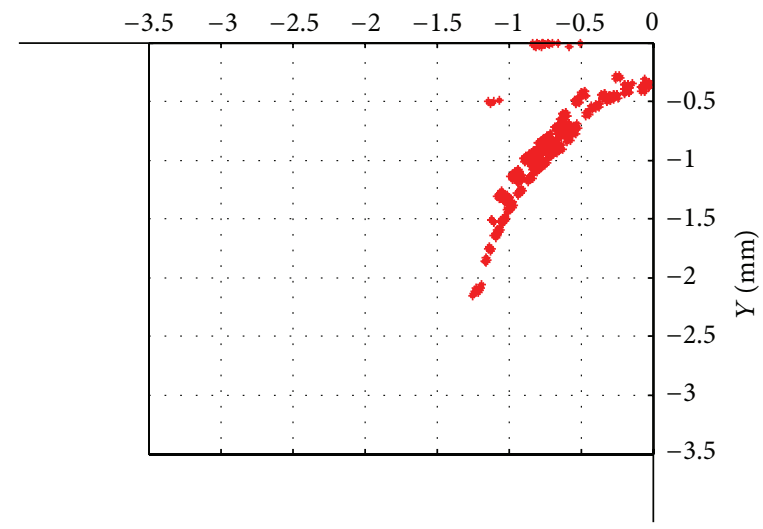

(c)

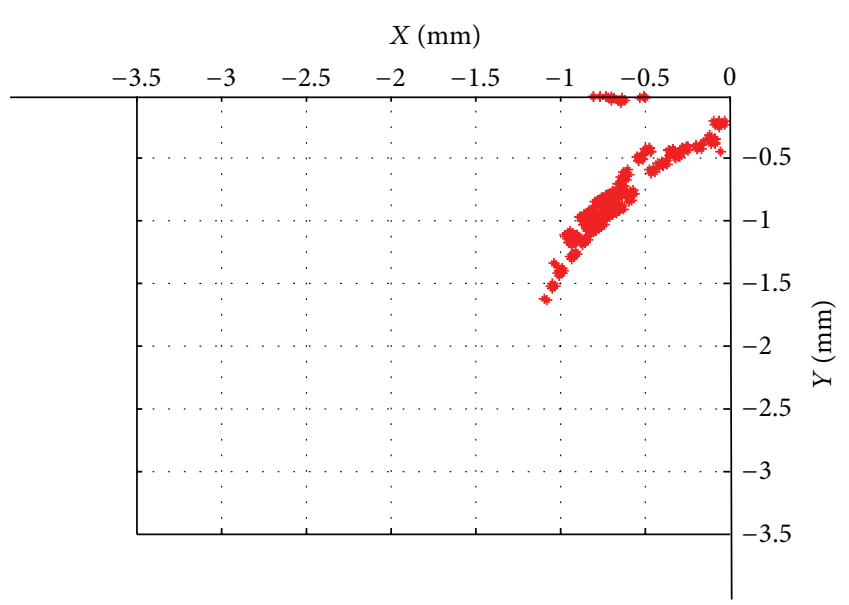

(b)

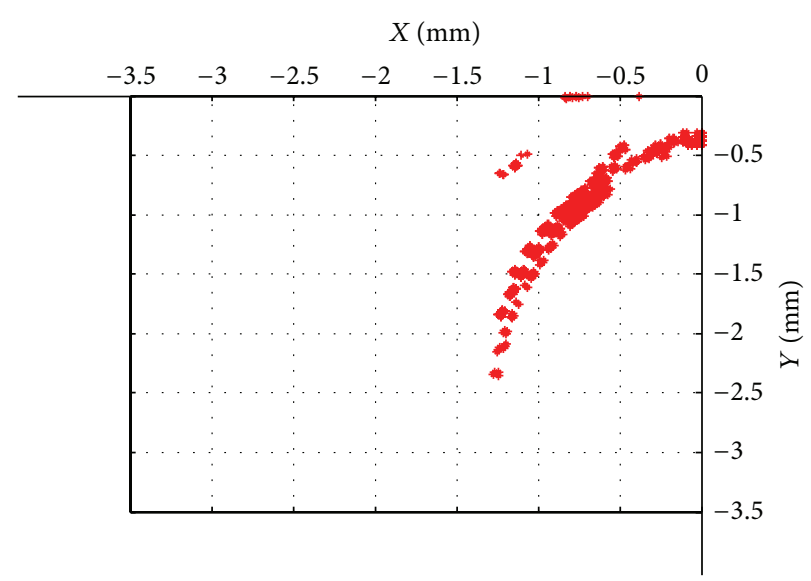

(d)

FIGURE 9: Simulated crack locations close to the middle plane by using different values of the effective plastic displacement coefficient to failure (a) $0.8 \mu \mathrm{m}$; (b) $0.6 \mu \mathrm{m}$; (c) $0.4 \mu \mathrm{m}$; and (d) $0.3 \mu \mathrm{m}$. 


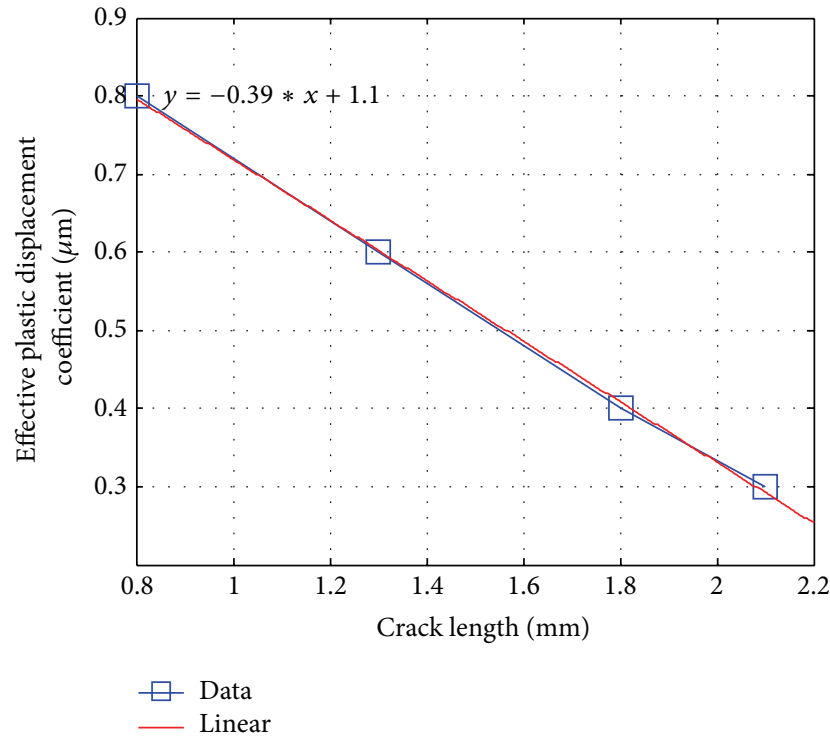

Figure 10: The relationship between the effective plastic displacement coefficient at the failure point and the cracking length $L$ along the circumference of the ellipse.

cracks were located at three regions: (1) along the thinner edge; (2) around $0.3-0.5 \mathrm{~mm}$ away from the thinner edge; and (3) around $0.8-1.6 \mathrm{~mm}$ away from the thinner edge. Comparing to the experimental crack images shown in Figure 8(b), the simulated results have successfully predicted the cracks generated at the location around $0.8-1.5 \mathrm{~mm}$ away from the thinner edge. However, the cracks observed 2.0-2.5 mm away from the thinner edge in experiments were not shown in the simulation.

Overall, the testing results showed that the simulated crack sizes and locations in the coupon models were roughly consistent with the experimental results. To the best of our knowledge, this is the first time the formation of cracks was simulated on an LSP processed airfoil-like structure. All JC plastic constants in the crack generation model were calibrated with experimental residual stress profiles in a thick plate based on the reasonable assumption that the plastic behaviors in two procedures, that is, residual stress development in thick plates and crack formation in airfoillike structures, are similar. The simulation of the residual stress profile in a thick plate consisted of two phases. In the first phase, the shock waves traveled through the component and then completely attenuated to the point where only elastic strain could continue. The second phase was the recovery process, in which no plastic deformation occurred. The duration selected for the first step was $4000 \mathrm{~ns}$ based on the convergence study. The calibrated JC plastic constants described the plastic behavior during the first $4000 \mathrm{~ns}$ of the LSP process. The crack generation model for airfoil coupons consisted of only one dynamic step, whose duration was also 4000 ns. Therefore, it is reasonable to apply the calibrated plastic constants to the crack generation model for airfoil coupons.

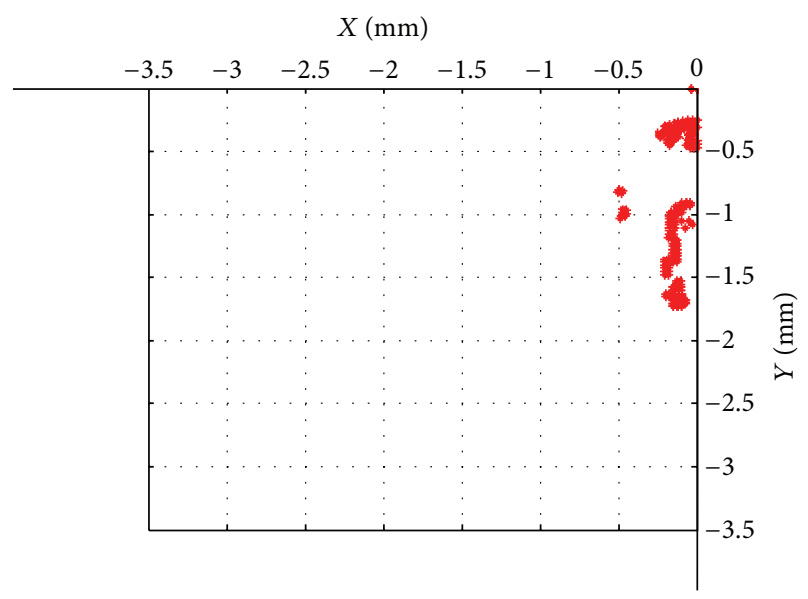

FIGURE 11: Simulated crack locations close to the middle plane for samples processed with small spots.

In this work, small discrepancies in crack locations between simulation and experimental results were observed when testing the model with a coupon processed with small laser spots; that is, the cracks located $2.0-2.5 \mathrm{~mm}$ away from the thinner edge in the experiments were not shown in the simulation (Figures 8(b) and 11). Possible explanations for this discrepancy are described as follows: (1) the material plastic constants used in this study were obtained through residual stress profile in a thick plate. Those material constants were then used for simulation of crack formation in thin airfoillike structures. In reality, there is a slight difference in plastic deformation between thick and thin samples. In thick plates, only compressive plastic deformation occurs. In thin sections, the shock wave may not have attenuated below the dynamic yield strength of the material before reaching the back surface. The reflected tensile shock wave from the free back surface is still capable of causing plastic strain. Both compressive and tensile plastic deformations occur. The JC plastic constants calibrated are compressive plastic properties. Therefore, the method of using JC compressive plastic constants to describe both tensile and compressive plastic behaviors in airfoil coupon model could potentially cause errors in the simulation. As the actual tensile yield strength is lower than the compressive yield strength, the use of compressive plastic properties to describe tensile plastic behaviors underestimates crack occurrence. (2) The fracture model is an important component in the model for crack generation simulation. The constants in damage initiation model were obtained from the literature, which means they could not be guaranteed for the absolute accuracy. For future research, the fracture model should be further improved by conducting more experiments to generate cracks by LSP process for calibrating the constants in damage initiation model. (3) It is considered that some cracks located in the microstructure of the material are highly dependent on its local material properties. This is particularly true at phase interfaces or grain boundaries. Therefore, structurescaled FEM models were not as accurate as we need in the experiment. A 3D microstructure-based model should 
be developed for use in future research. The use of this model would ensure that the grain and interphase boundary properties would be fully considered, which would increase the accuracy of the results. In order to construct such a model, it is important to characterize the microstructure properties experimentally. With the microstructure-based technique, it is expected that a more accurate damage model could be obtained. Since the microstructure-based properties, such as grain size and orientation and grain and interphase boundary properties, are not deterministic values, the probability of the crack generation could be obtained by assigning these properties in models based on their distributions.

\section{Conclusion}

In this study, a 3D FE model to simulate LSP-induced crack generation in Ti-6246 airfoil specimens was developed. This model was fully considered with the plastic and fracture behaviors at high strain rate by using both JC plastic and fracture models. The simulated crack sizes and locations in the airfoil coupon models are consistent with the experimental results. Therefore, FEM analysis could be applied to predict the generation and size of subsurface cracks, which helps to minimize the chance of producing subsurface cracks in LSP processing.

Although the initial results are promising, there is still plenty of room for further improvement of the current models. For example, the plastic model can be improved by considering the difference between tensile and compressive plastic behaviors. Likewise, there may be more experiments conducted to generate cracks by LSP process for the purpose of calibrating constants in the damage initiation model. In addition, a 3D microstructure-based model should be further developed by considering the grain and interphase boundary properties thoroughly.

\section{Conflict of Interests}

The authors declare that there is no conflict of interests regarding the publication of this paper.

\section{Acknowledgments}

This work was funded by the US Air Force Research Laboratory (AFRL/RXLP) under contract no. FA8650-08-C-5201. The authors would like to thank Mr. Siamack Mazdiyasni from AFRL/RXLP for his comments, suggestions, and support as they performed this research. They also would like to thank Dr. Allan H. Clauer and Mr. Richard D. Tenaglia at LSP Technologies, Inc., for the discussion and providing them with the LSP-processed samples and residual stress data.

\section{References}

[1] K. Ding and L. Ye, "FEM simulation of two sided laser shock peening of thin sections of Ti-6Al-4V alloy," Surface Engineering, vol. 19, no. 2, pp. 127-133, 2003.
[2] K. Ding and L. Ye, "Simulation of multiple laser shock peening of a 35CD4 steel alloy," Journal of Materials Processing Technology, vol. 178, no. 1-3, pp. 162-169, 2006.

[3] A. H. Clauer, Surface Performance in Titanium, Edited by J. K. Gregory, H. J. Rack, and D. Eylon, The Minerals, Metals \& Materials Society, Warrendale, Pa, USA, 1996.

[4] C. A. Lavender, S.-T. Hong, M. T. Smith, R. T. Johnson, and D. Lahrman, "The effect of laser shock peening on the life and failure mode of a cold pilger die," Journal of Materials Processing Technology, vol. 204, no. 1-3, pp. 486-491, 2008.

[5] W. Braisted and R. Brockman, "Finite element simulation of laser shock peening," International Journal of Fatigue, vol. 21, no. 7, pp. 719-724, 1999.

[6] H. K. Amarchinta, R. V. Grandhi, A. H. Clauer, K. Langer, and D. S. Stargel, "Simulation of residual stress induced by a laser peening process through inverse optimization of material models," Journal of Materials Processing Technology, vol. 210, no. 14, pp. 1997-2006, 2010.

[7] Q. Liu, S. A. Barter, P. K. Sharp et al., "Effect of internal cracking on fatigue life of 7050 aluminum alloy treated by laser shock processing," in Proceedings of the 11th International Conference on Fracture (ICF11 '05), pp. 6093-6098, Turin, Italy, March 2005.

[8] Q. Liu, S. A. Barter, and G. Clark, "Internal cracking during surface treatment of 7050- T74 aluminium alloy using laser shock peening," in Proceedings of the International Conference on Structural Integrity and Fracture (SIF '02), September 2002.

[9] Q. Liu, S. A. Barter, P. K. Sharp, and G. Clark, "Investigation on internal cracking of 7050 aluminium alloy induced by laser shock processing," DSTO Research Report, Defence Science and Technology Organisation (DSTO), Department of Defense, The Australian Government, 2004.

[10] A. F. M. Arif, "Numerical prediction of plastic deformation and residual stresses induced by laser shock processing," Journal of Materials Processing Technology, vol. 136, no. 1-3, pp. 120-138, 2003.

[11] Y. Hu, Z. Yao, and J. Hu, "3-D FEM simulation of laser shock processing," Surface and Coatings Technology, vol. 201, no. 3-4, pp. 1426-1435, 2006.

[12] P. Peyre, I. Chaieb, and C. Braham, "FEM calculation of residual stresses induced by laser shock processing in stainless steels," Modelling and Simulation in Materials Science and Engineering, vol. 15, no. 3, pp. 205-221, 2007.

[13] C. Yang, P. D. Hodgson, Q. Liu, and L. Ye, "Geometrical effects on residual stresses in 7050-T7451 aluminum alloy rods subject to laser shock peening," Journal of Materials Processing Technology, vol. 201, no. 1-3, pp. 303-309, 2008.

[14] H. K. Amarchinta, R. V. Grandhi, K. Langer, and D. S. Stargel, "Material model validation for laser shock peening process simulation," Modelling and Simulation in Materials Science and Engineering, vol. 17, no. 1, Article ID 015010, 2009.

[15] G. R. Johnson and W. H. Cook, "A constitutive model and data for metals subjected to large strains, high strain rate and high temperatures," in Proceedings of the 7th International Symposium on Ballistics, pp. 541-547, 1983.

[16] “ABAQUS user's manual,” version 6.11.

[17] G. Kay, "Failure modeling of titanium $6 \mathrm{Al}-4 \mathrm{~V}$ and aluminum 2024-T3 with the Johnson-Cook material model," Final Report DOT/FAA/AR-03/57, Office of Aviation Research, Federal Aviation Administration, 2003. 

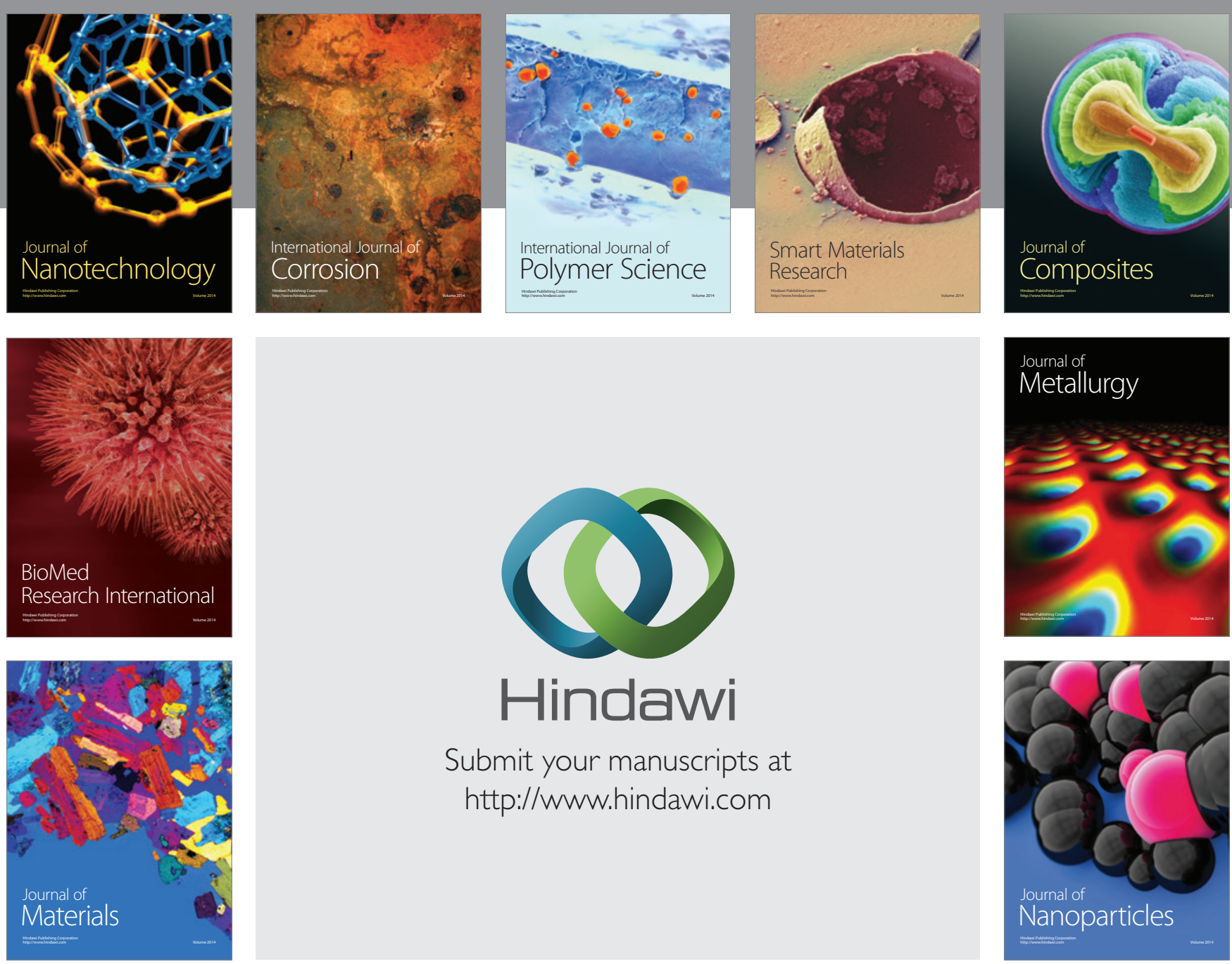

Submit your manuscripts at http://www.hindawi.com
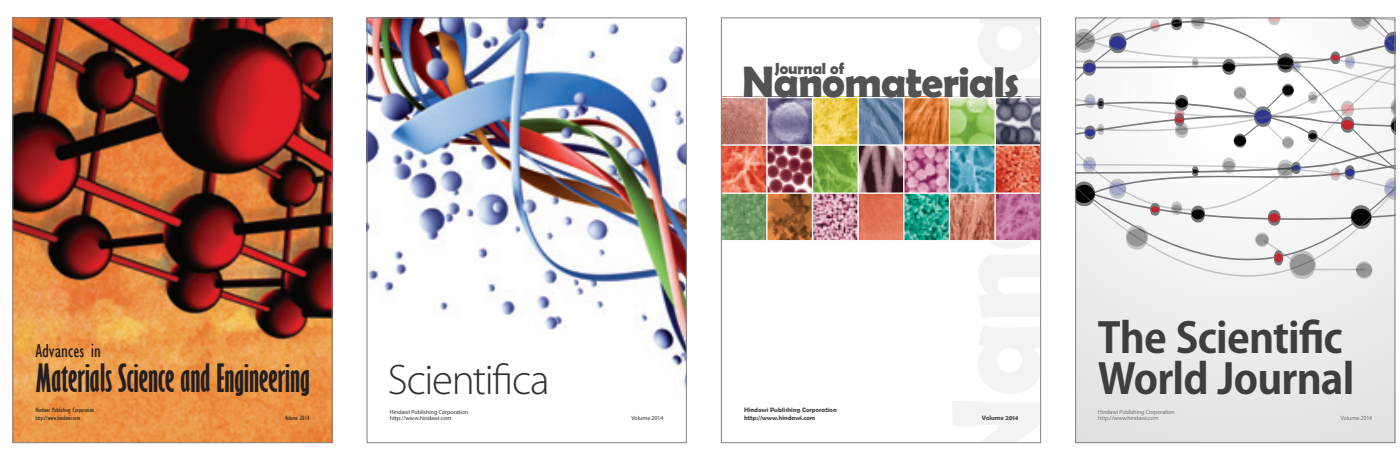

\section{The Scientific World Journal}
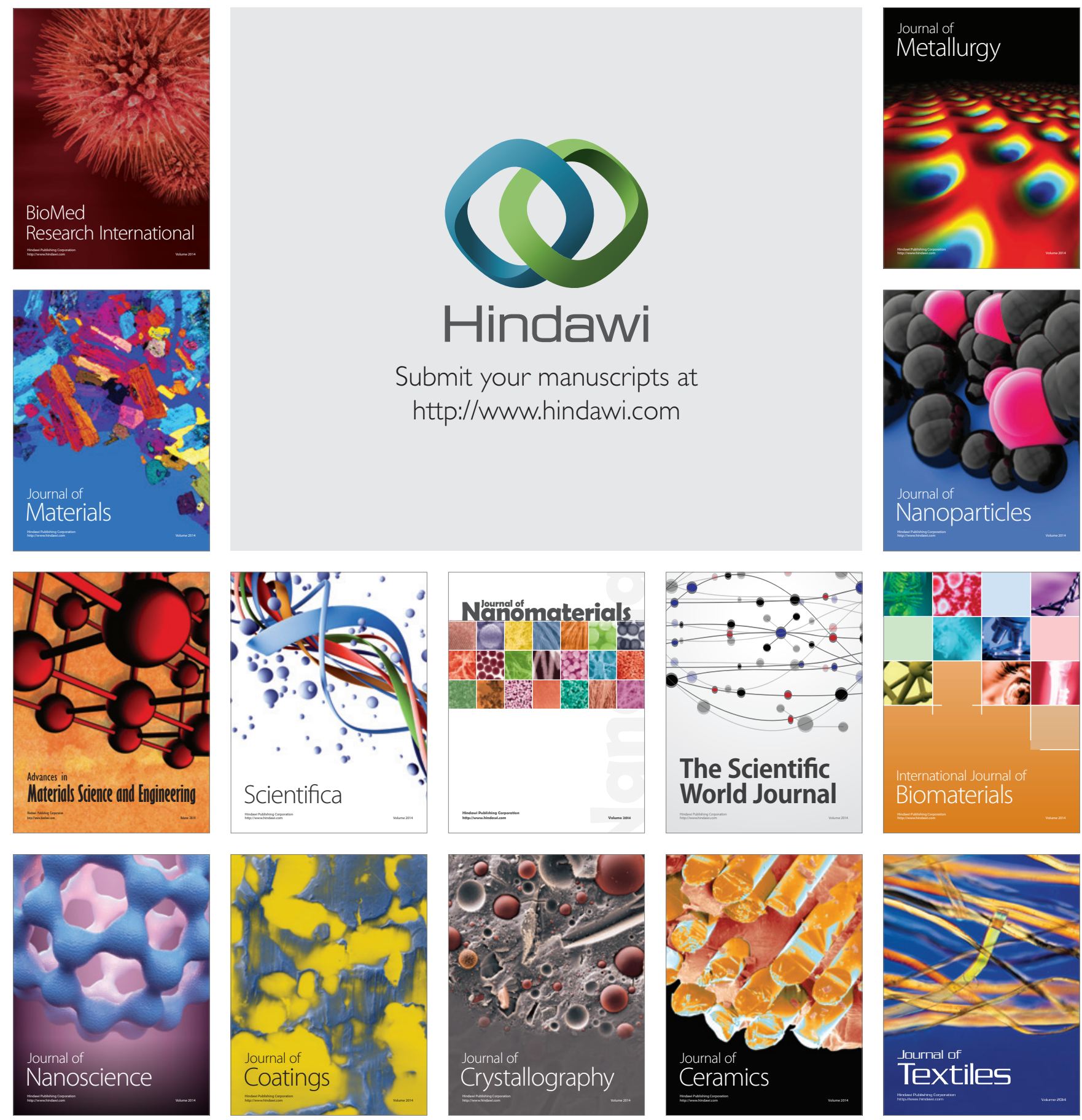